\title{
Mortalidad y factores pronósticos en pacientes hospitalizados por bacteriemia adquirida en la comunidad
}

\author{
P. SANZ CARABAÑA ${ }^{1}$, A. RAMOS MARTÍNEZ, A. ASENSIO VEGAS ${ }^{2}$, \\ $\mathrm{M}^{\mathrm{a}} \mathrm{J}$. GARCÍA NAVARRO, M. LINARES RUFO ${ }^{3}$
}

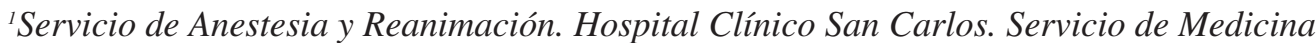
Interna. ${ }^{2}$ Servicio de Medicina Preventiva. ${ }^{3}$ Servicio de Microbiología. Hospital Universitario Puerta de Hierro. Madrid

\section{RESUMEN}

Introducción: La bacteriemia adquirida en la comunidad es un problema frecuente y de elevada mortalidad. El índice de Pitt, calculado en base a la temperatura, el estado mental, y las funciones circulatoria y respiratoria del paciente es una herramienta útil para conocer el pronóstico de pacientes con bacteriemia. Los objetivos fueron conocer la etiología de las bacteriemias adquiridas en la comunidad en nuestro entorno, el porcentaje de pacientes con tratamiento empírico incorrecto, y determinar la mortalidad y los factores independientes asociados con el fallecimiento del paciente, incluido el índice de Pitt.

Método: Estudio observacional de una cohorte de pacientes mayores de 18 años ingresados en un hospital terciario por una infección adquirida en la comunidad con bacteriemia. Veintiocho casos fueron desestimados por considerar el aislamiento bacteriano como contaminante.

Resultados: Se incluyeron 75 enfermos con un promedio de edad de 68,6 $\pm 18,2$ años, 38 mujeres (51\%). La mortalidad fue del 23\% [IC95\% $13,8-33,8]$. Las bacterias más frecuentemente aisladas en los hemocultivos fueron Escherichia coli, Staphylococcus aureus, estafilococos coagulasa negativo, Pseudomonas aeruginosa, Streptococcus viridans, enterococos y Klebsiella spp. La mortalidad de los pacientes con un tratamiento antibiótico empírico inadecuado (18\%, 3 pacientes) no fue inferior a los que recibieron un tratamiento empírico correcto en urgencias $(24 \%, 14$ pacientes, $\mathrm{p}=0,42)$. Los factores independientes pronósticos de mortalidad, fueron la baja concentración plasmática de albúmina, $\mathrm{OR}=5,17$ (IC 95\% 1,45-16,7) para cada descenso de $1 \mathrm{~g} / \mathrm{dl}$, el elevado índice de Pitt OR $=1,50$ (IC 95\% 1,01-2,24) para cada aumento de una unidad, y la elevada gravedad de la enfermedad de base medida por el índice de McCabe y Jackson OR = 5,08 ( IC 95\% 1,43-16,7).

Conclusiones: El tratamiento antibiótico empírico incorrecto no influyó en la mortalidad de pacientes con bacteriemia comunitaria. Los factores pronósticos de mortalidad fueron la concentración plasmática de albúmina, el índice de Pitt y la gravedad de la enfermedad de base medida por el índice de McCabe y Jackson.

PALABRAS CLAVE: Infecciones adquiridas en la comunidad. Bacteriemia. Agentes antibacterianos. Escherichia coli. Staphylococcus aureus. Albúmina. Mortalidad. Pronóstico.
MORTATILY AND PROGNOSTIC FACTORS IN PATIENTS ADMITTED WITH COMMUNITY-ACQUIRED BACTEREMIA

\section{ABSTRACT}

Background: Community-acquired bacteremia is a frequently clinical problem and is associated with a high mortality. The Pitt bacteremia score that is calculated using body temperature, mental status, and some respiratory and circulatory features is an useful tool in order to know the prognosis of partients with bacteremia. Objetives were to know microbial etiology of bacteremia in our community, percentage of patients that received an inadequate empiric antibiotic treatment and indepedent prognostic factors associated with mortality, including Pitt bacteremia score.

Method: Observational study of a cohort of patients over 18 years admitted at a tertiary hospital due to an infection with a communityacquired bacteremia. Twenty-eight cases were rejected because possible blood culture contamination.

Results: Seventy-five patients were included, the mean age was 68.6 \pm 18.2 years, 38 women $(51 \%$ ). Mortality rate was $23 \%$ [IC95\% 13.8 33.87. More frequent bacteria isolated were Escherichia coli, Staphylococcus aureus, coagulase negative staphilococci, Pseudomonas aeruginosa, Streptococcus viridans, enterococci and Klebsiella spp. Mortality in patients treated with an inadequate antibiotic therapy $(18 \%, 3$ patients) was not lower than in adequately treated patients (24\%, 14 patients, $p=0.42$ ). Indepedent prognostic factors related to mortality were serum albumin concentration, OR=5.17 (IC 95\% 1.45-16.7) for every downing step of $1 \mathrm{~g} / \mathrm{dl}$, the Pitt bacteremia score OR=1.50 (IC 95\% 1.01-2.24) for every unit increase, and a high score at McCabe and Jackson classification OR=5.08 ( IC 95\% 1.43-16.7)

Conclusions: An inadequate empiric antibiotic therapy was not associated with a worse vital prognosis. Indepedent prognostic factors related to mortality were serum albumin concentration, the Pitt bacteremia score, and the McCabe and Jackson classification.

KEY WORDS: Community-acquired infections. Bacteremia. Anti-bacterial Agents. Escherichia coli. Staphylococcus aureus. Albumins. Mortality. Prognosis.

Sanz Carabaña P, Ramos Martínez A, Asensio Vegas A, García Navarro M J, Linares Rufo M. Mortalidad y factores pronósticos en pacientes hospitalizados por bacteriemia adquirida en la comunidad. An Med Interna (Madrid) 2006; 23: 66-72. 


\section{INTRODUCCIÓN}

La incidencia de bacteriemia en pacientes ingresados en nuestro país se sitúa entre 14 y 21 casos por cada 1000 ingresos hospitalarios (1). La mortalidad de estos pacientes oscila entre el 14 y el $85 \%$, en función del foco infeccioso, del patógeno responsable, del lugar de adquisición y de la gravedad del enfermo (2-5). Aunque las bacteriemias adquiridas en la comunidad (BAC) presentan una gravedad menor que las nosocomiales, existen datos que sugieren que tanto su mortalidad como su frecuencia podrían verse incrementadas en los próximos años (6-9).

Conseguir prescribir un tratamiento antibiótico empírico correcto presenta grandes dificultades ante determinados tipo de pacientes que pueden estar infectados por un conjunto muy variado de especies bacterianas (9). El conocimiento precoz de las variables pronósticas de los pacientes que ingresan con BAC podría ser de ayuda en la prescripción de dicho tratamiento empírico (6).

El índice de Pitt, calculado en base a la temperatura, el estado mental, y las funciones circulatoria y respiratoria del paciente, es una herramienta útil para conocer el pronóstico de pacientes con bacteriemia (10-11). Aunque el pronóstico vital de las BAC ha sido objeto de varias publicaciones $(5,9,11)$, incluso en nuestro país $(6,12,13)$, en pocos trabajos se analiza el índice de Pitt como variable pronóstica en estos pacientes $(10,11)$.

El presente trabajo pretende conocer la etiología de las $\mathrm{BAC}$, el porcentaje de pacientes con tratamiento empírico incorrecto, y determinar la mortalidad y los factores independientes asociados con el fallecimiento del paciente, incluyendo entre ellos el índice de Pitt.

\section{MATERIAL Y MÉTODO}

Se realiza un estudio observacional de una cohorte de pacientes mayores de 18 años ingresados en un hospital terciario por una infección adquirida en la comunidad con bacteriemia. El centro donde se realizó el estudio es un hospital universitario con 500 camas y unos 13.000 ingresos anuales que atiende a una zona con un número elevado de residencias de ancianos.

Se incluyeron todos aquellos pacientes con sospecha de bacteriemia cuyos hemocultivos se hubieran obtenido durante las primeras 24 horas de estancia en el hospital. Para cada hemocultivo se obtienen $10 \mathrm{ml}$ de sangre que se divide en 2 fracciones de $5 \mathrm{ml}$ y se introducen en 2 frascos de $50 \mathrm{ml}$ cada uno para cultivo de microorganismos aerobios y anaerobios. Se utiliza el sistema automatizado Bactec NR 730 y se valora como positivo en la lectura automática el valor de 35 (punto de corte). Tras la confirmación del crecimiento de microorganismos en el hemocultivo, por parte del Servicio de Microbiología, se examinó al paciente y se recogió información de su historia clínica durante el ingreso para valorar los posibles factores de riesgo, el índice de Pitt y la evolución del paciente. El período del estudio incluyo desde enero a octubre de 1997 ambos incluidos. Se excluyeron aquellos pacientes con fungemia y aquellos otros que hubieran estado ingresados durante el mes previo con objeto de descartar a pacientes con bacteriemias de origen nosocomial.

Las especies de estafilococo coagulasa negativa, difteroides, Bacillus spp, Propionibacterium spp y micrococo fueron considerados contaminantes si crecieron sólo en los hemocultivos de una sola venopunción, el paciente no era portador de catéteres centrales o prótesis vasculares y no padecía una enfermedad que pudiera ser producida por estos patógenos. El resto de los casos fueron analizados minuciosamente por dos investigadores (PS y AR) para decidir su significación clínica. Durante el período de estudio se desestimaron 28 casos de hemocultivos positivos por ser considerados contaminantes. El tratamiento antibiótico empírico fue considerado apropiado si el paciente recibió al menos un fármaco activo frente a la bacteria aislada, la bacteria presentaba un antibiograma con sensibilidad al antibiótico administrado, y si este antibiótico fue administrado a las dosis e intervalos adecuados.

\section{VARIABLES ESTUDIADAS}

De cada uno de los pacientes se obtuvieron sus datos demográficos y procedencia (domicilio particular o residencia de ancianos); la presencia de enfermedades de base tales como insuficiencia renal crónica (creatinina $>1,7 \mathrm{mg} / \mathrm{dl}$ ), diabetes mellitus, neoplasias (exceptuando los tumores en remisión completa o las neoplasias cutáneas), enfermedad pulmonar obstructiva crónica, demencia, cardiopatía, hepatopatía crónica, infección por VIH; y la presencia de tratamientos y factores de riesgo previos a la bacteriemia tales como el tratamiento corticoideo (más de $15 \mathrm{mg}$ /día durante más de 15 días), tratamiento con inmunosupresores (en los últimos 3 meses), sondaje vesical, catéter central de larga duración, tratamiento antibiótico previo al ingreso, motivo de ingreso, y localización del foco infeccioso, gravedad de la enfermedad de base (según clasificación de McCabe y Jackson) (14). Fueron considerados pacientes con enfermedad últimamente mortal los enfermos con neoplasia, excepto si la neoplasia era cutánea, insuficiencia renal crónica, infección por VIH, cardiopatía terminal y hepatopatía crónica con ascitis. Los enfermos cuya enfermedad basal condicionase un desenlace inminente fueron considerados como portadores de enfermedad rápidamente mortal. Otras variables recogidas fueron la presencia de signos y síntomas clínicos tales como escalofríos, confusión mental, vómitos, hemorragia digestiva, dolor abdominal, disnea, pérdida de peso (más del 10\% en 6 meses), temperatura axilar al ingreso, frecuencia cardíaca, frecuencia respiratoria, tensión arterial sistólica, tensión arterial diastólica, además del índice de Pitt (Tabla I) (10). También se recogieron las siguientes determinaciones plasmáticas urea, creatinina, sodio, potasio, albúmina, bilirrubina, AST, ALT, lacticodeshidrogenasa, colesterol LDL, gasometría (en los pacientes con patología respiratoria), hemograma, neutropenia $\left(<1.000\right.$ leucocitos $\left./ \mathrm{mm}^{3}\right)$, INR, TTPA. Para caracterizar la infección se identificó la especie bacteriana aislada y su antibiograma, el origen del bacteriemia, los antibióticos recibidos, el tratamiento antibiótico empírico adecuado, si precisó ingreso en UCI, y tratamientos recibidos tales como la ventilación mecánica, hemodiálisis, empleo de drogas vasoactivas, distress respiratorio. Además se valoró la adecuación del tratamiento antibiótico realizado los días de estancia hospitalaria y si el paciente falleció.

\section{ANÁLISIS DE LOS DATOS}

Las variables categóricas se expresaron en porcentajes calculando el riesgo relativo (RR) y sus correspondientes inter- 
TABLA I

ÍNDICE GRAVEDAD DE BACTERIEMIA DE PITT (VERSIÓN DE 1998)

\begin{tabular}{ll}
\hline Temperatura & \\
$\leq 35{ }^{\circ} \mathrm{C}$ & 2 puntos \\
$35,1-36$ & 1 punto \\
$36,1-38,9{ }^{\circ} \mathrm{C}$ & 0 puntos \\
$39-39,9^{\circ} \mathrm{C}$ & 1 punto \\
$\geq 40^{\circ} \mathrm{C}$ & 2 puntos \\
Tensión arterial & \\
(a) Caída de $30 \mathrm{mmHg}$ en la TAS & \\
o de $20 \mathrm{~mm} \mathrm{Hg}$ en la TAD & 2 puntos \\
(b) Drogas vasoactivas & 2 puntos \\
(c) TAS < $90 \mathrm{mmHg}$ & 2 puntos \\
Ventilación mecánica & 2 puntos \\
Parada cardíaca & 4 puntos \\
Estatus mental & \\
Alerta & 0 puntos \\
Desorientado & 1 punto \\
Estuporoso & 2 puntos \\
Coma & 4 puntos \\
\hline
\end{tabular}

Todos los parámetros deben obtenerse 2 durante los dos días previos o durante el mismo día de la obtención de los hemocultivos. TAS: tensión arterial sistólica. TAD: tensión arterial diastólica

valos de confianza. Las variables continuas se expresaron con su media \pm desviación estándar. La hipótesis de normalidad fue evaluada mediante los test de Kolmogorov-Smirnov y de Shapiro-Wilk. Para evaluar las diferencias de las medias en el análisis univariante se emplearon la t de Student o el test no paramétrico de Mann-Whitney, si la normalidad no pudo ser asumida. Para comparar las variables categóricas se empleó la prueba exacta de Fisher, la prueba de la Chi cuadrado o la prueba de la Chi cuadrado para tendencias, si se sospechó un efecto gradiente dependiente. Además, mediante regresión logística múltiple se identificaron las principales factores independientes pronósticos de mortalidad hospitalaria. El modelo logístico fue construido desde un modelo máximo que incluía todas las variables independientes que en el análisis univariante mostraron una asociación estadística por debajo del nivel de significación 0,2. La variable principal era la mortalidad intrahospitalaria. Para el modelo final, se efectuó la selección de variables siguiendo una estrategia de paso a paso hacia atrás.

Para la identificación de los factores independientes que son pronósticos de mortalidad en los pacientes con bacteriemia de la comunidad se construyó un modelo multivariante. Dada la cantidad de potenciales factores pronósticos a estudio se seleccionaron en un primer paso variables a partir de tres diferentes grupos: variables provenientes de los análisis clínicos, variables de presentación clínica y localización del foco infeccioso, variables sobre las enfermedades de base, características clínicas y demográficas al ingreso, incluyendo el índice de Gravedad y el índice de Pitt. Cada uno de estos tres modelos previos se construyó a partir de todos aquellos factores de riesgo que se asociaron en el análisis univariante con un $\mathrm{p}<0,25 \mathrm{y}$ se seleccionaron. En un segundo paso todas las variables asociadas con una $p<0,25$ en cada uno de los tres grupos de variables se seleccionaron para construir un modelo máximo de factores pronósticos del que se fueron retirando variables paso a paso hacia atrás hasta obtener un modelo final con el mínimo número de variables significativamente asociadas a la mortalidad hospitalaria de forma independiente. Se consideró significación estadística un valor de $\mathrm{P}$ a dos colas menor de 0,05 .

\section{RESULTADOS}

Durante el periodo de estudio 75 enfermos ingresaron con BAC de los cuales 17 fallecieron (incidencia de mortalidad $23 \%$ [ $\left.\left.\mathrm{IC}_{95 \%} 13,8-33,8\right]\right)$. La cohorte de pacientes tenía una promedio de edad de $68,6 \pm 18,2$ años. Treinta y ocho pacientes $(51 \%)$ eran mujeres. Quince pacientes $(20 \%)$ provenían de residencias de ancianos. Las enfermedades crónicas más frecuentes fueron cardiopatía (28 pacientes, 37\%), demencia (14 enfermos, 19\%), diabetes mellitus (12 pacientes, 16\%), hepatopatía crónica (11 enfermos, $15 \%$ ) y neoplasia (11 pacientes, $15 \%$ ) (Tabla II). Respecto a la gravedad de la enfermedad de base, 3 pacientes $(4 \%)$ padecían una enfermedad rápidamente mortal, $16(21 \%)$ una enfermedad últimamente mortal y 56 (75\%) un padecimiento no mortal.

Las bacterias más frecuentemente aisladas en los hemocultivos fueron Escherichia coli, Staphylococcus aureus, estafilococos coagulasa negativo, Pseudomonas aeruginosa, Streptococcus viridans, enterococos y Klebsiella spp (Tabla III). En cuatro pacientes (5\%) se aislaron 2 especies bacterianas, ninguno de los cuales falleció. Las cocos grampositivos constituyeron el 51\% (40 aislados) frente al 49\% (39 aislados) de los bacilos gramnegativos. Entre los mayores de 70 años los gramnegativos constituyeron el 54\% de los aislados. En 13 ocasiones $(17 \%)$ la misma bacteria fue aislada en muestras relacionadas con el foco infeccioso primario. La mayoría de estos casos correspondían a $E$. coli aislado en orina (7 pacientes, $41 \%$ ). Cinco pacientes (7\%) además presentaban otra infección distinta a la que originó la bacteriemia consistentes en infección respiratoria, celulitis, infección del tracto urinario, meningitis aséptica (presuntamente viral) y candidiasis oral que afectaron a un paciente cada una respectivamente.

Las causas de fallecimiento fueron la progresión del cuadro séptico (12 pacientes, 71\%), el edema agudo de pulmón (2 pacientes, $12 \%$ ), el infarto agudo de miocardio (un paciente, $6 \%$ ), la obstrucción intestinal (un paciente, 6\%) y la progresión tumoral metastásica (un paciente, $6 \%$ )

Los síntomas y signos exploratorios más frecuentes figuran en la Tabla II. Destacó la asociación entre mortalidad y la pérdida de peso superior al 10\% en los seis meses previos $(\mathrm{p}=$ $0,073)$ o la presencia de úlceras por presión $(\mathrm{p}=0,043)$. En el análisis univariante se muestra que los pacientes fallecidos presentaron unos niveles mayores de urea y menores de leucocitos, plaquetas, albúmina y colesterol LDL (Tabla IV). No hubo diferencias relevantes en la gasometría y estudio de coagulación.

El origen respiratorio de la bacteriemia se asoció con una mayor mortalidad y el urinario con una mortalidad más baja (Tabla V). El 22\% de este último grupo correspondía a portadores crónicos de sonda vesical. Cinco pacientes presentaron endocarditis, dos (40\%) de ellas por $S$. aureus sensible a meticilina.

Los dos pacientes con neumonía bacteriémica por $P$. aeruginosa fallecieron, uno recibió empíricamente amoxicilina/clavulánico y el otro imipenem. Los dos pacientes con bacteriemia por Streptococcus intermedius también fallecieron (Tabla III), 
TABLA II

CARACTERÍSTICAS CLÍNICAS Y DEMOGRÁFICAS AL INGRESO DE LOS PACIENTES CON BACTERIEMIA COMUNITARIA

\begin{tabular}{|c|c|c|c|c|}
\hline & Fallecidos $n=17$ & Vivos $n=58$ & $R R(95 \% \mathrm{Cl})$ & $P$ \\
\hline Edad (años)* & $68,8 \pm 17,6$ & $68,6 \pm 18,5$ & - & 0,909 \\
\hline Sexo femenino & $8(47)$ & $30(52)$ & $1,16(0,5-2,7)$ & 0,735 \\
\hline Provenientes de residencia & $4(23)$ & $11(19)$ & $1,2(0,5-3,2)$ & 0,703 \\
\hline \multicolumn{5}{|l|}{ Tipo de enfermedad de base } \\
\hline Rápidamente mortal & $2(12)$ & $1(2)$ & $2,6(1,2-5,8) 1$ & 0,024 \\
\hline Últimamente mortal & $7(41)$ & $10(17)$ & & \\
\hline \multicolumn{5}{|l|}{ Enfermedad de base } \\
\hline Insuficiencia renal & $3(17)$ & $7(12)$ & $1,4(0,4-4,0)$ & 0,551 \\
\hline Diabetes mellitus & $3(17)$ & $9(15)$ & $1,1(0,4-3,3)$ & 0,833 \\
\hline Neoplasia & $5(29)$ & $6(10)$ & $2,6(1,1-6,0)$ & 0,052 \\
\hline EPOC 2 & $2(12)$ & $6(10)$ & $1,1(0,3-4,0)$ & 0,867 \\
\hline Infección por VIH & $0(0)$ & $2(3)$ & 0 & 0,595 \\
\hline $\mathrm{ACVA}^{3}$ & $3(17)$ & $5(9)$ & $1,8(0,6-4,9)$ & 0,257 \\
\hline Tratamiento corticoideo & $4(23)$ & $4(7)$ & $2,6(1,1-6,0)$ & 0,072 \\
\hline Tratamiento inmunosupresor & $2(12)$ & $5(9)$ & $1,3(0,4-4,5)$ & 0,695 \\
\hline Escalofríos & $3(19)$ & $27(46)$ & $0,3(0,1-1,1)$ & 0,085 \\
\hline Confusión & $7(41)$ & $21(36)$ & $1,2(0,5-2,7$ & 0,709 \\
\hline Disnea & $5(29)$ & $16(28)$ & $1,1(0,4-2,9)$ & 0,773 \\
\hline Vómitos & $2(12)$ & $18(31)$ & $0,4(0,1-1,5)$ & 0,098 \\
\hline Hemorragia digestiva alta & $3(17)$ & $2(3)$ & $3(1,3-7,1)$ & 0,073 \\
\hline Dolor abdominal & $6(35)$ & $19(32)$ & $1,2(0,5-2,9)$ & 0,722 \\
\hline Pérdida de peso (> $10 \%$ en 6 meses) & $3(18)$ & $2(3)$ & $3,0(1,3-7,1)$ & 0,073 \\
\hline Temperatura $\left({ }^{\circ} \mathrm{C}\right)$ & $38,4 \pm 1,1$ & $38,0 \pm 1,0$ & & 0,296 \\
\hline Frecuencia cardíaca por minuto & $100 \pm 29$ & $100 \pm 19$ & & 0,735 \\
\hline
\end{tabular}

${ }^{1}$ comparando enfermedad no mortal con rápidamente y últimamente mortal. ${ }^{2}$ EPOC: Enfermedad Pulmonar Obstructiva. ${ }^{3}$ Accidente cerebrovascular agudo. ${ }^{4}$ dosis equivalente de prednisona superior a $15 \mathrm{mg}$ durante más de 3 semanas.

pero la causa del fallecimiento fue edema agudo de pulmón en ambos casos.

Tres pacientes que murieron $(18 \%)$ habían recibido tratamiento antibiótico antes de ingresar (en 2 casos el germen aislado no era sensible a dicho antibiótico) y 12 enfermos (21\%) entre los supervivientes (en 8 casos sin cobertura antibiótica adecuada, $\mathrm{p}>0,05)$. En tres de estos casos el paciente ingresó sin tratamiento antibiótico alguno y en los 14 restantes la bacteria aislada era resistente al tratamiento prescrito. Los pacientes con cobertura inicial inadecuada padecían infección del tracto urinario (12 pacientes, 71\%), infección respiratoria (2 pacientes, 12\%), infección de la piel (1 paciente, $6 \%$ ), infección abdominal (1 paciente, $6 \%$ ) y bacteriemia primaria (1 paciente, $6 \%)$. La mortalidad en estos pacientes $(18 \%, 3$ pacientes) fue similar a los que recibieron un tratamiento empírico correcto en urgencias $(24 \%, 14$ pacientes, $\mathrm{p}=0,42)$.

Cinco pacientes que finalmente fallecieron habían ingresado en UCI $(29 \%)$ y $9(16 \%)$ de los supervivientes $(p=0,196)$. Los fallecidos presentaron más frecuentemente distress respiratorio del adulto $(35 \%$ frente a $10 \%$ en los supervivientes, $\mathrm{p}<0,001)$, ventilación mecánica (23\% frente al 5\%, p = 0,096), y el empleo de drogas vasoactivas (35\% frente al 7\%, p = 0,007).

Los principales factores independientes pronósticos de mortalidad, obtenidos mediante análisis multivariante, fueron la baja concentración plasmática de albúmina, el elevado índice de Pitt y la elevada gravedad de la enfermedad de base medida por el índice de McCabe y Jackson (Tabla VI).

\section{DISCUSIÓN}

Como en otros trabajos similares, la mayoría de los casos de BAC fueron secundarias a un foco infeccioso conocido. El origen mayoritario de la bacteriemia en las distintas series suele variar en función del tipo de pacientes que sean objeto del estudio. Así, en una serie de pacientes con BAC ingresados en UCI el número de pacientes con foco pulmonar, urinario o abdominal fue similar (6). Sin embargo, en pacientes con menor gravedad 
TABLA III

ETIOLOGÍA DE BACTERIEMIA COMUNITARIA

\begin{tabular}{|c|c|c|c|c|}
\hline Especie bacteriana & Fallecidos $(n=17)$ & Vivos $^{*}(n=58)$ & $R R(I C 95 \%)$ & $P$ \\
\hline Staphylococcus aureus & 5 & 10 & $1,7(0,7-3,9)$ & 0,285 \\
\hline Staphylococcus aureus meticilin-resistente & 0 & 2 & & 0,595 \\
\hline Staphylococcus epidermidis & 0 & 3 & $0,5(0,1-3,4)$ & 0,457 \\
\hline Staphylococcus epidermidis meticilin-resistente & 1 & 3 & & 0,651 \\
\hline Enterococcus sp & 0 & 3 & & 0,346 \\
\hline Streptococcus pneumoniae & 1 & 1 & $2,3(0,5-9,7)$ & 0,404 \\
\hline Streptococcus agalactiae & 0 & 1 & & 0,585 \\
\hline Streptococcus bovis & 0 & 1 & & 0,585 \\
\hline Streptococcus intermedius & 2 & 0 & $4,9(3,1-7,6)$ & 0.049 \\
\hline Streptococcus viridans & 1 & 3 & $1,1(0,2-6,4)$ & 0,651 \\
\hline Escherichia coli & 4 & 23 & $0,6(0,2-1,5)$ & 0,223 \\
\hline Klebsiella spp & 0 & 3 & & 0,457 \\
\hline Pseudomonas aeruginosa & 2 & 3 & $1,9(0,6-6,0)$ & 0,316 \\
\hline Salmonella typhimurium & 1 & 0 & $4,6(3,0-7,1)$ & 0,223 \\
\hline Enterobacter cloacae & 0 & 1 & & 0,585 \\
\hline Gemella morbillorum & 0 & 1 & & 0,585 \\
\hline
\end{tabular}

*En cinco pacientes se aislaron 2 especies distintas de bacterias Klebsiella pneumonia- Citrobacter freundii, Enterococcus faecium- Proteus mirabilis, Escherichia col-Enterococcus faecalis y Staphylococcus aureus-Streptococcus pyogenes.

TABLA IV

PARÁMETROS ANALÍTICOS AL INGRESO EN PACIENTES CON BACTERIEMIA ADQUIRIDA EN LA COMUNIDAD

\begin{tabular}{|c|c|c|c|}
\hline & Fallecidos $(n=17)$ & $\operatorname{Vivos}(n=58)$ & $P$ \\
\hline Urea $(\mathrm{mg} / \mathrm{dl})$ & $99 \pm 54$ & $66 \pm 49$ & 0,012 \\
\hline Creatinina (mg/dl) & $2,0 \pm 1,4$ & $1,8 \pm 1,2$ & 0,685 \\
\hline Sodio $(\mathrm{mEq} / \mathrm{l})$ & $140 \pm 12$ & $137 \pm 7$ & 0,610 \\
\hline Leucocitos ( $10^{9}$ cél/L) & $12,8 \pm 7,6$ & $16,7 \pm 7,9$ & 0,006 \\
\hline Cayados (109 cél/L) & $0,9 \pm 1,1$ & $1,0 \pm 1,6$ & 0,653 \\
\hline Neutropenia1 ( $<10^{9}$ leucocitos/L) & $2(12)$ & $3(5)$ & 0,316 \\
\hline Plaquetas $\left(10^{9} \mathrm{cél} / \mathrm{L}\right)$ & $153 \pm 114$ & $203 \pm 103$ & 0,024 \\
\hline Hemoglobina (g/dl) & $11,6 \pm 2,9$ & $12,5 \pm 2,3$ & 0,222 \\
\hline INR & $1,4 \pm 0,5$ & $1,6 \pm 1,5$ & 0,491 \\
\hline APTT (segundos) & $49 \pm 26$ & $47 \pm 40$ & 0,590 \\
\hline Actividad de protrombina (\%) & $65 \pm 17$ & $70 \pm 21$ & 0,320 \\
\hline $\mathrm{pH}^{*}$ & $7,38 \pm 0,1$ & $7,40 \pm 1,1$ & 0,286 \\
\hline $\mathrm{pO} 2 *(\mathrm{~mm} \mathrm{Hg})$ & $74 \pm 29$ & $70 \pm 14$ & 0,803 \\
\hline $\mathrm{pCO}^{*}$ (mm Hg) & $32 \pm 6$ & $33 \pm 6$ & 0,803 \\
\hline Bicarbonato (mEq/l) & $21 \pm 5$ & $23 \pm 6$ & 0,161 \\
\hline Albúmina sérica (g/dl) & $2,6 \pm 0,6$ & $3,4 \pm 0,7$ & 0,001 \\
\hline Bilirrubina (mg/dl) & $1,3 \pm 1,6$ & $1,5 \pm 2,3$ & 0,254 \\
\hline AST $(U / L)$ & $48 \pm 40$ & $69 \pm 107$ & 0,796 \\
\hline $\operatorname{ALT}(U / L)$ & $38 \pm 27$ & $74 \pm 137$ & 0,346 \\
\hline LDH (U/L) & $431 \pm 211$ & $422 \pm 136$ & 0,649 \\
\hline Colesterol LDL (mg/dl) & $105 \pm 34$ & $146 \pm 46$ & 0,003 \\
\hline
\end{tabular}

*30 pacientes. ${ }^{*} 60$ pacientes APTT. INR: razón normalizada internacional. 1RR (IC AL 95\%)=1,9 (0,6-6,0)

suele predominar un origen urinario (9). Aunque las bacteriemias de origen pulmonar suelen presentar un mal pronóstico, la relación coste-eficacia de los hemocultivos es cuestionada por algunos investigadores dado que con frecuencia no conlleva un cambio en el tratamiento empírico (15), en esta serie sólo en 2 de los pacientes con bacteriemia de origen respiratorio (12\%) la cobertura antibiótica inicial era inadecuada cifra inferior a la del resto de pacientes $(26 \%, \mathrm{p}=0,188)$.

En la mayoría de los trabajos sobre BAC, las bacterias grampositivas son las más frecuentemente aisladas $(5,16)$, aunque en hospitales de tamaño pequeño pueden predominar los bacilos gramnegativos (9). Los hospitales que atienden a una población más vulnerable como los portadores del VIH, diabéticos y pacientes en hemodiálisis suelen presentar también un predominio de los grampositivos en los casos de BAC (9). El incremento de pacientes con BAC por Staphylococcus epidermidis se ha relacionado con el empleo de catéteres centrales de larga duración (17). Este fenómeno sólo ocurrió en 
TABLA V

LOCALIZACIÓN DEL FOCO INFECCIOSO ORIGINARIO DE BACTERIEMIA DE ORIGEN COMUNITARIO

\begin{tabular}{lcccc}
\hline Origen de la bacteriemia & Fallecidos $(n=17)$ & Vivos $(n=58)$ & $R R(I C 95 \%)$ & $P$ \\
\hline Abdominal & $5(29)$ & $6(10)$ & $2,4(1,1-5,5)$ & 0,064 \\
Piel & $2(12)$ & $1(2)$ & $3,2(1,3-8,0)$ & 0,126 \\
Endocarditis & $0(0)$ & $5(9)$ & & 0,265 \\
Foco desconocido & $0(0)$ & $4(7)$ & & 0,266 \\
Aparato locomotor & $0(0)$ & $3(5)$ & & 0,457 \\
Aparato respiratorio & $8(47)$ & $9(16)$ & $3(1,4-6,6)$ & 0,011 \\
Urinario & $2(12)$ & $30(52)$ & $0,2(0,1-0,7)$ & 0,003 \\
\hline
\end{tabular}

TABLA VI

FACTORES PRONÓSTICOS DE MORTALIDAD HOSPITALARIA EN PACIENTES CON BACTERIEMIA ADQUIRIDA EN LA COMUNIDAD. ANÁLISIS MULTIVARIANTE

\begin{tabular}{lcccc}
\hline Factor pronóstico & coeficiente $B$ & $E E$ & OR $(95 \% \mathrm{Cl})$ & $P$ \\
\hline Albúmina $^{1} \mathrm{~g} / \mathrm{dl}$ & 1,642 & 0,645 & $5,17(1,45-16,7)$ & 0,011 \\
Índice de Pitt $^{2}$ & 0,407 & 0,203 & $1,50(1,01-2,24)$ & 0,045 \\
Gravedad enfermedad basNal $^{3}$ & 1,625 & 0,647 & $5,08(1,43-16,7)^{*}$ & 0,012 \\
\hline
\end{tabular}

${ }^{1}$ por cada descenso en $1 \mathrm{~g} / \mathrm{dl}^{2}$ por cada aumento en una unidad del índice. ${ }^{3}$ por cada aumento de categoría de riesgo

uno de nuestros pacientes. Determinadas circunstancias aumentan el riesgo de que una endocarditis sea la causa de una bacteriemia por $S$. aureus como son la existencia de valvulopatía, la adquisición comunitaria, la infección por VIH y la ausencia de foco primario evidente (11). Dos de nuestros pacientes con BAC por S. aureus (12\%) padecían endocarditis, cifra inferior al $21 \%$ encontrada por Chang et al (11).

Como ha sido encontrado en otros trabajos similares $E$. coli, $P$. aeruginosa y Klebsiella spp fueron son los bacilos gramnegativos más frecuentemente aislados $(5,18)$. También la frecuencia de BAC polimicrobianas fue similar a la de otras series publicadas $(5,6)$.

La mortalidad en nuestros enfermos (23\%) es algo superior a la de series como la de Larka et al (5) que alcanzó el 14\%. La mayoría de nuestros pacientes $(71 \%)$ fallecieron por progresión de la infección. Cabe destacar que la edad no se ha asociado con la mortalidad en el presente estudio, pero eso puede ser debido a la elevada edad de la cohorte con un promedio de 68 años y donde el $70 \%$ de los pacientes eran mayores de 60 años. El predominio de gramnegativos (E. coli) en ancianos podría ser la causa de una mejor respuesta al tratamiento.

Aunque se ha sugerido que los pacientes con BAC que no presentan fiebre tendrían una menor respuesta inflamatoria y que esto ha sido asociado a una mayor mortalidad (19), en este trabajo los pacientes que fallecieron tuvieron una temperatura superior al ingreso que los supervivientes $(p=0,296)$. La posible administración de antipiréticos que no fue analizada podrían estar relacionadas con este resultado.

En el análisis univariante se evidenciaron cifras mas elevadas de urea entre los que fallecieron. Otros autores también han encontrado una relación entre la mortalidad y la función renal (5). También una cifra de plaquetas inferior se ha relacionado con la mortalidad como aconteció en esta serie (7). Ambos parámetros se podrían deber a una inestabilidad hemodinámica y una mayor respuesta inflamatoria respectivamente, en los pacientes que finalmente fallecieron.

Nuestros pacientes con neumonía bacteriémica presentaron una mortalidad del 47\% ( $\mathrm{p}=0,011)$. Similares resultados han obtenido otros autores lo que puede ser debido a la insuficiencia respiratoria que se añade al síndrome séptico $(5-8,20)$. Llamó la atención que $S$. pneumoniae estuviera implicado sólo en 2 pacientes, siendo la mayoría de las neumonías bacteriémicas causadas por S. aureus (7 casos, $41 \%$ ) y por gramnegativos (3 pacientes, $18 \%$ ). La edad avanzada de nuestros paciente y su frecuente procedencia de residencias de ancianos podría justificar la etiología de las neumonías en estos pacientes.

Así mismo, varios estudios coinciden en un mejor pronósticos para las bacteriemia de origen urinario como en este trabajo lo que sugiere una menor gravedad, la elevada concentración de los antibiótico en el sistema urinario, la escasa propensión hacia la formación de abscesos y las escasas repercusiones funcionales que presentan en comparación con las infecciones pulmonares o abdominales pudieran ser las responsables de un mejor pronóstico vital (5-8).

La BAC polimicrobiana se ha asociado a una mayor mortalidad $(5,17)$, sin embargo ninguno de los cuatro pacientes estudiados es esta serie falleció. Al igual que otros autores hemos observado una mortalidad elevada en los casos de BAC por Pseudomonas spp (40\%), aunque en el presente trabajo no alcanzó significación estadística, quizás por un representar un número escaso de casos. Esta misma observación puede hacer a los casos infectados por $S$. pneumoniae (5). También la mortalidad por $S$. aureus ha sido elevada (29\%, p =0,326). Los dos casos de BAC por SARM sobrevivieron al ingreso. Sin embargo, otros autores han atribuido una mayor mortalidad a los pacientes con bacteriemia por SARM en base a una hipotética mayor virulencia, un posible retraso en la aplicación del tratamiento correcto en algunos casos y a una menor actividad antibacteriana sobre SARM de la vancomicina en relación a los $\beta$ lactámicos (21). El único microorganismo que se asoció con la mortalidad en el análisis univariante fue $S$. intermedius, en ambos casos el paciente falleció por edema agudo de pulmón con una celulitis y una infección abdominal, respec- 
tivamente. Por ello, no se puede atribuir la muerte a este agente de modo directo.

Los casos con cobertura antibiótica empírica inadecuada (17 pacientes, 23\%) fue ligeramente superior a la de otros trabajos similares $(6,7,22,23)$. Destacando un porcentaje menor en las series de enfermos críticos lo que suele determinar una tratamiento empírico más amplio $(6,23)$. A pesar de ello, no se objetivó una relación directa la cobertura antibiótica empírica incorrecta y la mortalidad. Aunque otros autores tampoco han hallado una relación independiente con la mortalidad $(7,9,24)$,otros trabajos, especialmente los basados en enfermos críticos han señalado a esta variable como muy relacionada con la supervivencia del paciente $(6,22,25)$. Tal vez, el número elevado de pacientes con sepsis de origen urinario entre los que tuvieron un tratamiento empírico inadecuado pudo influir en este hecho. Por otra parte se debe destacar que la magnitud de la serie estudiada no es grande y por tanto su valor para enjuiciar el papel pronóstico de un tratamiento empírico correcto puede ser limitado.

El índice de Pitt estuvo asociado de modo independiente con la mortalidad $(10,11)$. Este índice puede reflejar adecuadamente la respuesta inflamatoria a la infección al incluir variables sobre la temperatura, tensión arterial, nivel de conciencia y función pulmonar. Otros autores también han relacionado la hipotensión arterial y el shock con la mortalidad en pacientes con BAC (9). Es posible que las variables que en el se recogen se adapten mejor a los enfermos con bacteriemia que otros índices de gravedad más complejos como el APACHE (10).

La albúmina plasmática, que podría reflejar el estado nutricional previo y el efecto de la infección sobre el catabolismo de proteínas, se ha asociado con la mortalidad de pacientes con bacteriemia en otros estudios $(5,6,18)$. La albúmina junto a la clasificación de McCabe podrían reflejar el estado basal del paciente. Por tanto, en esta serie tanto variables asociadas con el estado basal del paciente como las relacionadas con la gravedad de la respuesta a la infección (evaluado mediante el índice de Pitt) estuvieron relacionadas con la mortalidad del paciente.

No obstante la no identificación de otros factores pronósticos en nuestra cohorte debe realizarse con cautela dado que el tamaño muestral del estudio no garantiza una potencia suficiente para identificar otros factores pronósticos que estén asociados con menor intensidad a la mortalidad.

\section{Bibliografía}

1. Martínez Luengas F y Grupo colaborador para el estudio de bacteriemias. Bacteriemia en 6 hospitales españoles. Med Clin (Barc) 1986; 86: 221-32.

2. Weinstein MP, Towns ML, Quartey SM, Mirrett S, Reimer LG, Parmigiani $\mathrm{G}$ et al. The clinical significance of positive blood cultures in the 1990s: A prospective comprehensive evaluation of the microbiology, epidemiology, and outcome of bacteremia and fungemia in adults. Clin Infect Dis 1997; 24: 584-602.

3. Lizarralde E, Gutiérrez A, Martínez P, Ibarmia J, de la Villa FM. Pronóstico de las bacteriemias adquiridas en la comunidad ingresadas en un Servicio de Medicina Interna. An Med Interna (Madrid) 2005; 22: 108-13.

4. Madsen KM, Schonheyder HC, Kristensen B, Sorensen HT. Secular trends in incidence and mortality of bacteraemia in a Danish county 1981-1994. APMIS 1999; 107: 346-52

5. Larka BL, Saintd S, Chenowetha C, Zemencukd JK, Lipskyb BA, Plordeb JJ. Four-year prospective evaluation of community-acquired bacteremia:Epidemiology, Microbiology, and patient outcome. Diagn Microbiol Infect Dis 2001; 41: 15-22.

6. Valles CJ, Rello J, Ochagavia A, Garnacho J, Alcalá MA. Communityacquired bloodstream infection in critically ill adult patients. Chest 2003; 123: 1615-24

7. Casariego E, Abraira V, Corredoira JC, Alonso MP, Rodríguez A, López MJ et al A predictive model for mortality of bloodstream infections:Bedside analysis with the Weibull function. J Clin Epidemiol 2002; 55: 563-72.

8. Ruiz-Giardin JM, Noguerado A. Bacteriemias. An Med Interna (Madrid) 2005; 22: 105-7.

9. Mylotte JM, Kahler L, McCann C. Community-acquired bacteremia at a teaching versus a nonteaching hospital: Impact of acute severity of illness on 30-day mortality Am J Infect Control 2001; 29: 13-9.

10. Chow JW, Yu VL. Combination antibiotic therapy versus monotherapy for gram-negative bacteremia: A commentary. Int $\mathrm{J}$ Antimicrob Agents 1999; 11: 7-12.

11. Chang FY, MacDonald BB, Peacock JE, Musher DM, Triplett P, Mylotte JM, et al. A Prospective Multicenter Study of Staphylococcus aureus Bacteremia Incidence of Endocarditis, Risk Factors for Mortality, and Clinical Impact of Methicillin Resistance. Medicine 2003; 82: 322-32.

12. Rodríguez FJ. Bacteriemias adquiridas en la comunidad. Rev Clin Esp 1997; 197: Suppl 5:10-4.

13. Cisterna R, Cabezas V, Gómez E, Busto C, Atutxa I, Ezpeleta C. Bacteriemias adquiridas en la comunidad. Rev Esp Quimioter 2001; 14: 369-82.

14. McCabe WR, Jackson GG. Gram-negative bacteremia. I. Etiology and ecology. Arch Intern Med 1962; 110: 847-64.

15. Campbell SG, Marrie TJ, Anstey R, Dickinson G, Ackroyd-Stolarz S. The contribution of blood cultures to the clinical management of adult patients admitted to the hospital with community-acquired pneumonia: A prospective observational study. Chest 2003; 123: $1142-50$

16. Noguerado A, Ruiz JM, Pizarro A, Méndez J, la Hulla F, Fernández M et al. Análisis de factores pronósticos de mortalidad de las bacteriemias y fungemias en un Hospital Universitario. Evolución en 10 años. Rev Clín Esp 2001; 201: 122-129.

17. Pittet D, Li N, Wenzel RP.Association of secondary and polymicrobial nosocomial bloodstream infections with higher mortality. Eur J Clin Microbiol Infect Dis 1993; 11: 813-9.

18. Diekema DJ, Pfaller MA, Jones RN, Doern GV, Winokur PL, Gales AC, et al. Survey of bloodstream infections due to gram-negative baci1li: frequency of occurrence and antimicrobial susceptibility of isolates collected in the United States, Canada, and Latin America for the SENTRY Antimicrobial Surveillance Program, 1997. Clin Infect Dis 1999; 29: 595-607.

19. Sonnenblick M, Carmon M, Rudenski B, Friedlander Y, Van Dijk JM. Septicemia in the elderly: incidence, etiology and prognostic factors. Isr J Med Sci 1990; 26: 195-9.

20. Reyes WJ, Brimiouilles S, Vincent JL. Septic shock without documented infection: an uncommon entity with a high mortality. Intens Care Med 1999; 25: 1267-70.

21. Melzer M, Eykyn SJ, Gransden WR, Chinn S. Methicillin-Resistant Staphylococcus aureus More Virulent than Methicillin-Susceptible S. aureus? A Comparative Cohort Study of British Patients with Nosocomial Infection and Bacteremia. Clin Infect Dis 2003; 37: 1453-60.

22. Pazos R, Fernandez R, Paz I, Tinajas A, Canton I, Abel V, Gonzalez R, et al. Factores pronósticos de bacteriemias: un estudio prospectivo. An Med Interna (Madrid) 2001; 18: 415-20.

23. Ibrahim EH, Sherman G, Ward S, Fraser VJ, Kollef MH. The influence of inadequate antimicrobial treatment of bloodstream infections on patients in the ICU setting. Chest 2000; 118: 146-55.

24. Amit M, Pitlik SD, Samra Z, Konisberger H, Drucker M, Leibovici L. Bacteriemia in patients without known underlying disorders. Scand J Infect Dis 1994; 26: 605-609.

25. Leibovici L, Samra Z, Konigsberger H, Drucker M, Ashkenazi S, Pitlik SD. Long-term survival following bacteremia or fungemia. JAMA 1995; 274: 807-12. 